\title{
Premature differentiation of nephron progenitors and dysregulation of gene pathways critical to kidney development in a model of preterm birth
}

\section{Aleksandra Cwiek}

University of Virginia Children's Hospital

Masako Suzuki

Albert Einstein College of Medicine

Kimberly deRonde

University of Virginia Children's Hospital

Mark Conaway

University of Virginia Health System

Kevin Bennett

Washington University in St. Louis

\section{Samir El Dahr}

Tulane University

\section{Kimberly Reidy}

Children's Hospital at Montefiore

Jennifer Charlton ( $\nabla$ jrc6n@virginia.edu )

University of Virginia Children's Hospital

\section{Research Article}

Keywords: preterm birth, neonatal morbidity, kidney health

Posted Date: April 26th, 2021

DOI: https://doi.org/10.21203/rs.3.rs-444923/v1

License: (1) (1) This work is licensed under a Creative Commons Attribution 4.0 International License. Read Full License 


\section{Abstract}

Preterm birth is a leading cause of neonatal morbidity. Survivors have a greater risk for kidney dysfunction and hypertension. Little is known about the molecular changes that occur in the kidney of individuals born preterm. Here, we demonstrate that mice delivered two days prior to full term gestation undergo premature cessation of nephrogenesis, resulting in a lower glomerular density. Kidneys from preterm and term groups exhibited differences in gene expression profiles at 20- and 27-days postconception, including significant differences in expression of fat-soluble vitamin-related genes. Kidneys of the preterm mice exhibited decreased proportions of endothelial cells and a lower expression of genes promoting angiogenesis compared to the term group. Kidneys from the preterm mice also had altered nephron progenitor subpopulations, early Six2 depletion, and altered Jag1 expression in the nephrogenic zone, consistent with premature differentiation of nephron progenitor cells. In conclusion, preterm birth alone was sufficient to shorten the duration of nephrogenesis and cause premature differentiation of nephron progenitor cells. These candidate genes and pathways may provide targets to improve kidney health in preterm infants.

\section{Introduction}

Significant advances have been made in neonatal medicine over the past three decades. Neonates are now considered viable in the $22^{\text {nd }}$ week of gestation ${ }^{1,2}$. These preterm neonates form nearly half of their lifetime supply of nephrons in an ex utero environment ${ }^{3}$. Nephrogenesis occurs through a complex interaction between the branching ureteric bud and the metanephric mesenchyme. In humans, a selfrenewing population of nephron progenitor cells in the metanephric mesenchyme niche forms new nephrons until 32-36 weeks ${ }^{3-5}$. These progenitor cells self-renew and differentiate, forming structures from the glomerulus to the collecting duct. However, common ex utero exposure to nephrotoxic medications, hypoxia, and hyperoxia, is unfavorable for nephrogenesis $s^{6,7}$ and is associated with poor short-term outcomes in the kidney, such as acute kidney injury 8,9 .

Preterm neonates who graduate from neonatal intensive care also have a greater risk for poor long term kidney outcomes such as chronic kidney disease (CKD) ${ }^{10-13}$. Adults born preterm have an increased risk for developing CKD, detectable as early as childhood ${ }^{10-12,14}$. These children are also at increased risk of high blood pressure and hypertension during childhood ${ }^{14-20}$. Although growing evidence suggests a significant connection between preterm birth and CKD, it is challenging to study the direct effect of preterm birth on long-term kidney outcomes. A great deal is unknown due to highly variable monitoring practices and a lack of evidence-based guidelines for monitoring kidney health after discharge from the neonatal intensive care unit.

Early histologic changes provide clues to the pathogenesis of kidney disease in primates and humans born preterm ${ }^{21,22}$. The nephrogenic zone, the niche for the progenitor cell population and immature glomerular structures, is significantly smaller in the preterm baboons ${ }^{22}$. Similarly, the nephrogenic zone 
is present for a shorter duration in the preterm humans ${ }^{4}$. Abnormal glomeruli are observed in the superficial cortex in both of these groups. Many of the glomeruli exhibit a cystic Bowman's space and a shrunken tuft. In a case series of adolescents and adults born preterm who underwent a kidney biopsy for clinical indications, the major histologic finding was secondary focal segmental glomerulosclerosis ${ }^{23}$.

Several animal models of preterm birth have been developed, but research on the effect of preterm birth on the kidney is limited due to species-dependent kidney development and the resources necessary to conduct long-term studies in large animal models. Although baboons have a long gestational window with nephrogenesis complete prior to birth ${ }^{24}$, consistent with humans, the resources required to maintain a preterm baboon neonatal intensive care unit are prohibitive. Mouse, rat, and rabbit models have also been used to study preterm birth ${ }^{25,26}$, but the natural window of postnatal nephrogenesis is different in each species. In a novel mouse model published in 2012, Stelloh et al, ${ }^{18}$ examined the effect of preterm birth on the kidneys of mice delivered two days before the end of gestation. By early adulthood, the mice developed CKD and exhibited higher blood pressures, albuminuria, and lower glomerular filtration rates.

Here, we used this same preterm mouse model to examine the histologic and molecular changes in the kidney that occur shortly after birth, to identify pathways that may be therapeutically targeted to prevent CKD associated with preterm birth. We found that nephrogenesis in preterm mice terminated a full day earlier than in term mice. Preterm birth led to early differentiation of nephron progenitors and decreased podocyte and endothelial cell proportions. There was dysregulation of several gene pathways critical in kidney health, such as fat-soluble vitamins, angiogenesis, and immune cells.

\section{Methods}

\section{Animals}

All animal experiments were approved at the University of Virginia by the Institutional Animal Care and Use Committee in agreement with the National Institutes of Health Guide for the Care and Use of Laboratory Animals. The study is reported in accordance with ARRIVE guidelines. Timed pregnant CD-1 dams were purchased from Charles River Laboratory (Wilmington, MA). The preterm group was comprised of pups born by Cesarean section at 18 days post-conception (18 dpc). The dams were euthanized by cervical dislocation before the Cesarean section as previously published ${ }^{18,27}$. Following a midline incision, the uterine horns were removed and the offspring were carefully expelled from their amniotic sacs. The placenta was separated from each pup using forceps to compress the umbilical cord. The pups were dried and gently stimulated with cotton-tipped applicators. They were placed in an incubator at $37^{\circ} \mathrm{C}$ and provided oxygen (95\%) until their skin became pink. The preterm pups were placed with a foster mother after her own pups were removed and euthanized. Acepromazine maleate (AceproJect $10 \mathrm{mg} / \mathrm{ml}$, Henry Schein Animal Health) with a final concentration of $75 \mathrm{mg} / \mathrm{ml}$ was added to the water of all mothers to reduce the risk of neglection for a total of 48 hours in both groups. The term group of control animals comprised pups that were delivered vaginally at $20 \mathrm{dpc}$. The pups were euthanized daily from 18-27 dpc in the preterm group ( $\mathrm{n}=3-11 /$ group) and from 20-27 dpc in the term 
group ( $n=3-16 /$ group). The kidneys were collected and weighed, and the right kidney was stored in RNAlater $^{\text {TM }}$ Stabilization Solution (ThermoFisher Scientific, AM7020) for gene expression analyses.

\section{Histology and immunohistochemistry.}

The left kidney was bisected along the transverse plane and immersion fixed in $10 \%$ buffered formalin for 24 hours, embedded in paraffin, and sectioned at $4 \mu \mathrm{m}$. Kidney sections were deparaffinized with xylene, followed by a rehydration procedure in a descending series of ethanol concentrations. Sections were stained with Periodic acid-Schiff (PAS) to determine glomerular area and density in both the preterm and term groups at $27 \mathrm{dpc}$. Biotinylated Lotus tetragonolobus lectin (LTL) (Vector Laboratories, Burlingame, CA, USA, B1325, 1:50 dilution) was used to identify mature proximal tubules to determine cessation of the nephrogenic zone and identify immature structures (vesicles along with S-shaped and comma-shaped structures). To perform LTL staining, endogenous peroxidase was quenched by submersion of the slides in $30 \%$ hydrogen peroxide in methanol for 30 minutes. The kidney sections were treated for 30 minutes by enzymatic digestion by proteinase $\mathrm{K}$ in $0.1 \mathrm{M} \mathrm{Na}-\mathrm{PO}_{4}$ buffer, then with biotinylated LTL for 1 hour. Following incubation, the sections were washed twice for 5 minutes in phosphate buffer and incubated in Vectastain $A B C$ reagent for 30 minutes. The sections were then incubated with 3,3'-diaminobenzidine (DAB), counterstained with a $0.5 \%$ methylene blue solution, and dehydrated.

Kidney sections stained with PAS and LTL were imaged using Grundium Ocus with a 20x objective (৫) Grundium Ltd 2019) for the following assessments. The nephrogenic zone was assessed in LTL-stained kidneys in both the preterm and term cohort from 18-26 dpc. The nephrogenic zone was considered depleted when the mesenchymal cap cells were absent and the LTL positive cells of the proximal tubules reached the capsule. The presence of immature glomerular structures (vesicle, S-shape, comma-shaped structures) was determined in LTL-stained tissues in both preterm and term cohorts from 18-26 dpc. To measure the glomerular area and density of the kidney sections at $27 \mathrm{dpc}$, the cortex was segmented from the medulla on PAS-stained sections using Amira software (Thermo Scientific) to measure cortical area. The capillary tuft of each glomerulus was outlined in a midsagittal section to calculate the glomerular area. Glomerular density was reported as the number of glomeruli/cortical area and was normalized to body weight.

To identify podocytes, kidney sections from term and preterm animals at $20 \mathrm{dpc}$ were exposed to a $0.45 \%$ methanol quench, antigen retrieval with $10 \%$ citrate buffer, blockade of endogenous biotin activity with avidin/biotin blocking kit (Vector Laboratories), and $100 \mu \mathrm{l}$ of goat serum in $1 \mathrm{ml}$ of $\mathrm{NaPO}_{4}$ for 1 hour. A monoclonal anti-human Wilms Tumor 1 (WT1) mouse antibody (Dako, M3561, 1:100 dilution) was applied to the sections and incubated overnight at $4^{0} \mathrm{C}$. The sections were washed in phosphate buffer then incubated with secondary goat anti-mouse antibody (Bio-Rad, 170-6516, 1:200 dilution) for 1 hour. Following an avidin-biotin complex to amplify the target antigen signal (Vector Laboratories, Avidin/Biotin Blocking Kit, SP-2001), the fixed antibody was detected with DAB. The sections were counterstained with methylene blue. Kidney sections from the preterm and term groups at 20 and 22 dpc were prepared similarly to detect Six2 and Jag1 (Jagged 1). The tissue was blocked with donkey serum in 
$1 \mathrm{ml}$ of $5 \%$ BSA in PBS for 1 hour, followed by primary antibody application, and incubated overnight at $4^{0} \mathrm{C}$. Both Six2 rabbit polyclonal antibody (Proteintech, 11562, 1:200 dilution) and Jag1 rabbit polyclonal antibody (Santa Cruz, 8303, 1:200 dilution) were co-stained with an anti-cytokeratin mouse antibody (Sigma Aldrick, c2562, 1:200 dilution) to outline the ureteric bud structures. Sections were incubated with secondary donkey anti-rabbit and anti-mouse antibodies for 1 hour (AlexaFluor 594 and 488, 1:250 dilution). Images were obtained using Microscope Leica Microsystems CMS (Leica DM1000 LED) and DMC6200 digital camera.

\section{RNA extraction:}

RNA was extracted from the whole right kidney from the mice at $18 \mathrm{dpc}(\mathrm{n}=3), 20 \mathrm{dpc}$ (preterm $\mathrm{n}=3$, term $n=3$ ) and $27 \mathrm{dpc}$ (preterm $n=3$, term $n=6$ ) with TRIZOL (Invitrogen ${ }^{\mathrm{TM}}, 15596018$ ) and cleaned with RNeasy MinElute Cleanup Kit (Qiagen, 74204) with RNase-Free DNase Set (Qiagen, 79254). Eluted RNA was reconstituted with RNase-free water to a final volume of $50 \mu \mathrm{l}$ and stored in $-80^{\circ} \mathrm{C}$ until analysis. RNA concentration was quantified and quality was assessed with a NanoDrop ${ }^{\mathrm{TM}}$ One/OneC Microvolume UVVis Spectrophotometer (Thermo Fisher Scientific).

\section{RNA-Sequencing:}

Gene expression profiles of the mouse kidneys from the preterm and term groups were evaluated by RNASeq on oligo-dT enriched mRNA using NEBNext ${ }^{\circledR}$ Ultra ${ }^{T M}$ RNA Library Prep Kits (non-directional) for Illumina, following manufacturer protocols. RNA sequencing was performed using RNA extracted. The generated libraries were sequenced on NovaSeq600 with 150 bp paired-end sequencing. The quality of the sequences was assessed by FASTQC (http://www.bioinformatics.babraham.ac.uk/projects/fastqc/), and the library qualities were evaluated with $\mathrm{RSeQC}^{28}$ before the analysis. The obtained sequences were aligned to the mouse genome (mm10) with GenCode vM15 as a reference annotation using STAR aligner $^{29}$. We counted the aligned reads per transcript for further analysis. The sequencing statistics are provided in Supplementary Table S1. We used DEseq2 to identify the differentially expressed genes ${ }^{30}$. Genes with at least a two-fold difference with a false discovery rate (FDR) adjusted $p<0.05$ were considered differentially expressed genes. The Gene Ontology enrichment analyses were implemented by the ClusterProfiler Bioconductor package at significance level $q<0.05$. Each analysis was adjusted for the sex of the offspring. All statistical analyses were performed using R (version 4.0.2).

\section{Estimating cell subtype proportion of mouse kidneys from the RNA-seq data:}

The cell subtype proportions were estimated based on the gene expression profiles of $20 \mathrm{dpc}$ preterm and term RNA-seq with a publicly available single-cell RNA-Seq (scRNA-seq) dataset of $20 \mathrm{dpc}$ mouse kidney CD-1 mice (GSM2473317) ${ }^{31}$ using CIBERSORTx algorithm ${ }^{32}$. The scRNA-seq data were re-analyzed before the CIBERSORTx analysis with Seurat to identify clusters of cell types and the expression status of the clusters ${ }^{33}$. The cell subtypes of each cluster were identified by the expression status of the marker genes $^{31}$. Identified clusters with the gene expression status of each cell were used to create a signature 
expression profile. The generated signature profile was used to estimate fractions of cell subtypes from each RNA-seq dataset. We used the S-mode batch correction with 100 permutations to generate the signature profile. The cell cycle of each cell was estimated by the CellCycleScoring function of Seurat using the expression status of cell-cycle specific genes, and pseudotime analysis was performed to assess the development time of the nephron progenitors proximal tubules and distal tubules by Monocle 3 algorithm ${ }^{34}$.

\section{Candidate gene analysis}

We applied a candidate gene approach to analyze the RNA-seq data. The candidate gene list provided in

Supplementary Table S2 was generated a priori from the literature. The genes of interest were categorized into three groups: (1) important in kidney development, (2) specific to a nephron segment, or (3) involved in kidney development pathways. We developed 30 categories within these three groups. Genes were categorized based on the location of gene expression and each gene was assigned to only one category. We ranked the categories by the number of genes differentially expressed between the preterm and term groups. The gene expression of the preterm group was compared to the term group at both 20 and 27 dpc. We corrected for multiple comparisons using a false discovery rate of 0.05 . The $p$-values were derived from the two-sample t-test with pooled variance. Point estimates and $95 \%$ confidence interval $(95 \% \mathrm{Cl})$ were calculated for each gene. Multivariate analysis without adjustments was conducted on each category of genes to compare point estimates from the preterm and term groups at both 20 and 27 dpc.

\section{Quantitative reverse transcription (RT)-PCR:}

To verify the RNA-Seq findings, we assessed the gene expression status using reverse-transcription, followed by quantitative PCR. A total of $1 \mu \mathrm{g}$ of RNA was reverse-transcribed with MultiScribe ${ }^{\text {TM }}$ Reverse Transcriptase (Thermo Fisher Scientific) to synthesize first-strand CDNA. PCR mixtures ( $10 \mu \mathrm{l})$ containing cDNA template equivalent to $10 \mathrm{ng}$ total RNA, $5 \mu \mathrm{M}$ of gene-specific primer pairs, and PowerUp SYBR Green Master Mix (Thermo Fisher Scientific) were prepared and subject to PCR amplification using the CFX Connect Real-Time PCR Detection System (Bio-Rad). The gene-specific primer pairs for Acy3, Crabp1, Cyp24a1, Cyp27b1, Pfdn4, Wnt11, Slc34a1, Nog, Etv5, and S14 were designed with Primer 3 (http://bioinfo.ut.ee/primer3-0.4.0). The primer sequences and conditions of PCR amplification are listed in Supplementary Table S3. S14, (a housekeeping gene commonly used in studies of kidney development), was used as a reference gene to assess the relative changes in gene expression ${ }^{35}$. Data are reported as medians and interquartile ranges and analyzed using a t-test with a $p$-value $<0.05$ being considered statistically significant.

\section{Results}

The body and kidney weights of the preterm and term groups are shown in Figure 1. The preterm group had a higher body weight than the term group after $23 \mathrm{dpc}$ (Figure 1a). While absolute kidney weight was 
similar between the preterm and term groups (Figure 1b), the kidney weight/body weight (KW/BW) was smaller in the preterm cohort on both 20 and $27 \mathrm{dpc}$ (Figure 1c). Glomerular density was lower in the preterm group compared to the term group at $27 \mathrm{dpc}$ (Figure 1d).

\section{Premature cessation and differentiation of nephron progenitors following preterm birth}

We identified immature nephron structures using the LTL-stained tissue samples to assess postnatal nephrogenesis. During nephrogenesis, the nephrogenic zone was present until $23 \mathrm{dpc}$ in the preterm group and until $24 \mathrm{dpc}$ in the term group (Figure 2a, b). After the cap mesenchymal cells were no longer present, the immature structures including vesicles, comma-shaped bodies, and S-shaped bodies, remained identifiable until $24 \mathrm{dpc}$ in the preterm group and $25 \mathrm{dpc}$ in the term group (Figure 2c). The progenitor cell population was further characterized by the histologic expression of Six 2 and Jag 1 in the nephrogenic zone. Six $2^{+}$cells were present in the cap mesenchyme in the term group at $20 \mathrm{dpc}$, but were absent in the preterm group at $20 \mathrm{dpc}$. There was no expression of Six 2 in the cap mesenchyme of either the term or preterm groups at $22 \mathrm{dpc}$. Jag1 was expressed in the preterm and term groups at 20 and 22 $\mathrm{dpc}$, but the location of the Jag1 was more prominent just under the capsule in the preterm group, where the cap mesenchyme had been depleted (Figure 2d). Together, these data indicate that animals born preterm have premature termination of nephrogenesis. In addition, there were fewer WT1+ cells in Sshaped bodies in the preterm group than in the term group (Figure $2 e$ and $f$ ).

\section{Transcriptional alterations in the kidney are present just after birth in preterm mice}

Transcriptional alterations were assessed at two time points, (early, 18-20 dpc and later: $27 \mathrm{dpc}$ ), using RNA-Seq. We performed an unsupervised analysis of the kidneys at $18 \mathrm{dpc}$ and 20 and $27 \mathrm{dpc}$ in the preterm and term groups. At the early time point (18 and $20 \mathrm{dpc}$ ), the expression profiles clustered into three groups (Figure 3a). The $18 \mathrm{dpc}$ group had distinct gene expression alterations compared to the groups at $20 \mathrm{dpc}$, as expected. A principal component analysis (PCA) indicated that a sexual dimorphism strongly contributed to PC2 (17\% variance) (Figure 3b). Therefore, we further evaluated the gene expression profiles both with and without adjustment for sex. We identified 39 and 38 differentially expressed genes (DEGs) between preterm and term groups at $20 \mathrm{dpc}, 760$ and 657 DEGs between $18 \mathrm{dpc}$ and preterm at $20 \mathrm{dpc}$, and 660 and 583 DEGs between $18 \mathrm{dpc}$ and term at $20 \mathrm{dpc}$, without and with adjustment for sex. The overlapped DEGs without and with adjustment for sex were 28 (preterm and term at $20 \mathrm{dpc}), 607$ (18 dpc and preterm at $20 \mathrm{dpc})$, and 566 (18 dpc and term at $20 \mathrm{dpc}$ ), respectively.

The complete lists of DEGs are shown in Supplementary Table S4-S6. To assess the biological functions of the DEGs, we performed gene ontology (GO) enrichment analyses using an R software package, clusterProfiler ${ }^{36}$. The vitamin D-related pathways were enriched in the top $G 0$ terms with and without adjusting for sex (Figure 3c-d), suggesting that the transcriptional alterations occur early. Changes were observed in the preterm group two days after birth.

At $27 \mathrm{dpc}$, the preterm and term groups did not fully segregate in the hierarchical clustering analysis (Figure 3e). However, PCA showed a clear distinction between preterm and term in PC1 (34\% variance). 
We observed segregation by sex in PC2 (20\% variance) (Figure $3 \mathrm{f})$, similar to the analysis at $20 \mathrm{dpc}$. We identified 703 and 558 DEGs between preterm and term kidneys at $27 \mathrm{dpc}$, without and with adjustment for sex. The complete lists of DEGs in each comparison are shown in Supplementary Table S7. While the number of DEGs was larger than at $20 \mathrm{dpc}$, there were no significant $\mathrm{GO}$ terms after adjusting for sex. Without adjusting for sex, the top GO term in the analysis of DEGs was small molecular catabolic processes (Figure 3g).

\section{Fewer endothelial cells and podocytes with alterations of nephron progenitor cells in preterm kidneys}

We investigated the relative proportion of cell subtypes in preterm and term groups at $20 \mathrm{dpc}$ using deconvolution of bulk gene expression (Figure $4 a$ ). We found 14 clusters of cell types in the scRNA-seq data, with the expression of 11845 genes in 9865 cells (Figure 4b). Both the proportion of endothelial cells and podocytes were lower in the preterm as compared to the term group (Figure 4a). The Cited $1^{+}$ nephron progenitors (NP1, Figure 4c) were decreased, and Sfrp2 ${ }^{+}$nephron progenitors (NP2, Figure 4d) were increased in preterm mice (Figure 4a). Sfrp2 is a target of the Wnt4 signaling pathway in kidney development ${ }^{37}$, a key pathway for nephron progenitor differentiation ${ }^{38}$. Together with the premature depletion of Six2 and increased Jag1 expression in the nephrogenic zone, these results suggest that the nephron progenitors prematurely differentiate in the preterm kidney.

\section{Alterations in angiogenesis and immune cell pathways}

The top three differential gene expression pathways at $20 \mathrm{dpc}$ were angiogenesis (Figure 5a), components of the glomerular basement membrane, and the renin-angiotensin system. Forest plots of all of the pathways are included in Supplementary Figure S3. The angiogenesis pathway and seven individual genes within the category (Aplnr, Cdh5, Nos3, Pecam1, Gpihhbp1, Efnb2, Flt1) had Cl's that indicated lower gene expression in the preterm group compared to the term group (Figure $5 \mathrm{a}$ ). In the glomerular basement membrane category, six genes (Lama5, Col4a6, Fras1, Col4a2m Col4a1, Col4a4) had Cl's indicating lower expression in the preterm group. The exception was Lama4 which had a $\mathrm{Cl}$ indicating higher expression in the preterm group. Three genes (Anpep, Agtr1-alpha, Cma1) included in the renin-angiotensin pathway had Cl's that indicated lower expression in the preterm group and three genes (NIn, Agtr1-beta, Agt) that had higher expression in the preterm group. None of the differences in individual genes reached statistical significance using the adjusted $\mathrm{p}<0.05$.

At $27 \mathrm{dpc}$, the top three pathways of differently expressed genes included immune cell designation (Figure $5 b)$, proximal tubule, and renin-angiotensin pathway. The category of immune cell designation and nine of the sixteen genes within this pathway had Cl's that indicated higher gene expression in the preterm group (Cd52, Ccl12, Fcgr3, Fcer1g, Laptm5, C1qc, Irf7, Coro1a, Lyz2) as compared to the term group. However, none of the individual genes reach statistical significance using the adjusted $p<0.05$. In the proximal tubule pathway, seven of the 25 genes were differentially expressed. Four genes (Gsta2, Hdc, $S / c 34 a 1, P d z k 1)$ had Cl's indicating lower expression in the preterm group and three genes (S/c5a2, S/c5a1, S/c3a1) had Cl's indicating higher expression in the preterm group (Supplementary Fig. S3). The 
genes Pdzk1, Gsta2, and S/c34a1, were significantly lower in the preterm group compared to the term group, using the adjusted $p<0.05$ (Table 1 ). The renin-angiotensin cascade pathway had six differentially expressed genes, with three genes with a $\mathrm{Cl}$ indicating a lower expression in the preterm group (Anpep, Enpep, and Ace2), and three genes had $\mathrm{Cl}$ indicating higher expression in the preterm group (Agtr1-beta, Ren 1 and Ren2). Ren1, and Ren2 were significantly greater in the preterm group using the adjusted $\mathrm{p}<0.05$ (Table 1).

Podocyte gene expression was examined at 20 and $27 \mathrm{dpc}$ because deconvolution analysis showed fewer podocytes at $20 \mathrm{dpc}$. At $20 \mathrm{dpc}$, four genes had Cl's indicating a lower expression in the preterm group (Nphs1, Plce1, Lmx1b, and Magi2). At $27 \mathrm{dpc}$, four genes had a Cl indicating a lower expression in the preterm group (WT1, Hs3st6, Plce1, and Foxd2). However, differential expression of these genes did not reach the threshold for significance.

Table 1. Statistically significant differentially expressed genes at $27 \mathrm{dpc}$ using the adjusted $\mathrm{p}$ value of $<0.05$.

\begin{tabular}{|l|l|l|l|l|}
\hline $\begin{array}{l}\text { Gene } \\
\text { symbol }\end{array}$ & $\begin{array}{l}\text { Estimate (higher in preterm } \\
\text { mice) }\end{array}$ & $\begin{array}{l}\text { Standard } \\
\text { error }\end{array}$ & $\begin{array}{l}95 \% \\
\text { interval }\end{array}$ & confidence \\
\hline Rgs5 & -0.272 & 0.039 & $(-0.365,-0.179)$ & $0.0002^{*}$ \\
\hline Ren2 & -0.445 & 0.067 & $(-0.603,-0.287)$ & $0.0003^{*}$ \\
\hline Ren1 & -0.445 & 0.067 & $(-0.603,-0.287)$ & $0.0003^{*}$ \\
\hline Etv4 & -0.283 & 0.044 & $(-0.388,-0.178)$ & $0.0004^{*}$ \\
\hline Slc5a2 & -0.175 & 0.028 & $(-0.242,-0.108)$ & $0.0004^{*}$ \\
\hline Etv5 & -0.307 & 0.052 & $(-0.430,-0.183)$ & $0.0006^{*}$ \\
\hline Mgp & -0.522 & 0.089 & $(-0.732,-0.312)$ & $0.0006^{*}$ \\
\hline
\end{tabular}

\begin{tabular}{|l|l|l|l|l|}
\hline $\begin{array}{l}\text { Gene } \\
\text { symbol }\end{array}$ & $\begin{array}{l}\text { Estimate (lower in preterm } \\
\text { mice) }\end{array}$ & $\begin{array}{l}\text { Standard } \\
\text { error }\end{array}$ & $\begin{array}{l}\text { 95\% Confidence } \\
\text { Interval }\end{array}$ & p-value \\
\hline Pdzk1 & 0.293 & 0.029 & $(0.224,0.361)$ & $\begin{array}{l}<0.0001 \\
*\end{array}$ \\
\hline Gsta2 & 0.879 & 0.101 & $(0.639,1.118)$ & $0.0001^{*}$ \\
\hline Slc34a1 & 0.330 & 0.049 & $(0.214,0.447)$ & $0.0003^{*}$ \\
\hline Igf2 & 0.503 & 0.086 & $(0.299,0.707)$ & $0.0006^{*}$ \\
\hline Cldn5 & 0.214 & 0.039 & $(0.123,0.306)$ & $0.0009^{*}$ \\
\hline Wnt11 & 0.278 & 0.056 & $(0.146,0.410)$ & $0.0016^{*}$ \\
\hline
\end{tabular}

\section{Discussion}

In this study, we demonstrate that preterm birth in mice is sufficient to induce early cessation of nephrogenesis by premature differentiation of the nephron progenitor cells. In an unbiased analysis, we 
observed dysregulation of the expression of genes related to fat-soluble vitamin metabolism at both 20 and $27 \mathrm{dpc}$. In a candidate gene approach, the pathway of angiogenesis was downregulated in the preterm mice at $20 \mathrm{dpc}$ and immune cells were upregulated at $27 \mathrm{dpc}$. Significantly, preterm birth alters the expression of genes related to proximal tubules and genes involved in the renin-angiotensin pathway. Mice born preterm emulate many of the characteristics observed after preterm birth in humans and the preterm mouse should serve as an essential model to dissect the molecular mechanisms connecting preterm birth to kidney disease and hypertension.

In humans, nearly $60 \%$ of nephrogenesis occurs in the third trimester ${ }^{39,40}$. Preterm birth in humans leads to early cessation of nephrogenesis and accelerated maturation of nephrons. Little is known about the fate of the progenitor pool, the signals that determine cessation of nephrogenesis, or why the duration of nephrogenesis varies between species. Hartman et al ${ }^{41}$ reported a consistent end to murine nephrogenesis at $24 \mathrm{dpc}$ in CD-1 mice. In the current study, the cap mesenchyme was consistently depleted by $23 \mathrm{dpc}$ in the preterm group, indicating cessation happened a day earlier than in the term group. A loss of one day of nephrogenesis is significant in mice because nearly $20 \%$ of glomeruli are created on the last day of nephrogenesis ${ }^{42}$. The premature end of nephrogenesis and lower glomerular density in the preterm group at $27 \mathrm{dpc}$ in this study are also consistent with the original findings of this model published by Stelloh et $\mathrm{al}^{18}$, which showed a $24 \%$ lower glomerular number in the preterm group.

There are several mechanisms that could lead to the premature cessation of nephrogenesis in this model, including the loss or premature differentiation of the self-renewing population of progenitor cells or decreased branching of the ureteric bud. Although reduced branching of the ureteric bud can reduce nephron number, this is less likely playing a role in this model because the change in environment is late in gestation, when the majority of branching is completed ${ }^{43}$. Our data support premature differentiation of the self-renewing population of progenitor cells as the primary mechanism leading to early cessation of nephrogenesis. We observed the loss of Six2 and an altered location of Jag1 protein expression in the nephrogenic zone of the preterm group at $20 \mathrm{dpc}$. The NP1 cell population was also significantly lower in the preterm kidney, but the NP2 population was higher. The NP1 population expressed the highest level of Cited 1 (Figure 4c) and Six2 (Supplementary Fig. S4). The NP2 population had a lower expression of Cited 1 (Figure 4c), but retained Six2 expression (Supplementary Fig. S4) with a higher expression of Lhx1, Jag1, and Sfrp2, indicating a more differentiated cell type in NP2. Using pseudotime trajectory analysis, we further examined these changes in the populations (Supplementary Figure S2). Most cells in the NP1 population that decreased in the preterm were in G1 of the cell cycle. Most cells in the NP2 population were in S or G2, suggesting they were proliferating (Supplementary Table S8). Together, this suggests that these NP1 cells may represent a early progenitor population with a slow turnover ( $\left.\mathrm{Cited}^{+}{ }^{+}, \mathrm{Six}^{+}\right)$, while NP2 may represent a more differentiated but proliferating population (Cited 1 , Six $2^{+}$, Sfrp $^{+}$), as previously described $^{38,44,45}$. The pseudo-trajectory analysis also suggested that the NP2 population represents nephron progenitor cells between NP1 and more differentiated cells (Supplementary Figure S2). Together, these data suggest preterm birth leads to depletion of multipotent $\left(\mathrm{Cited}^{+}\right.$, Six $\left.2^{+}\right)$progenitor cells, leading to early nephron cessation. Further work is necessary to understand the signaling that leads to 
premature differentiation of the nephron progenitors, including the metabolic factors, changes in stromal signaling, or alterations in the epigenetic regulation of these cells after birth.

We also identified alterations in several key kidney developmental pathways in the preterm group at 20 $\mathrm{dpc}$, including perturbations in angiogenesis and lower endothelial cell proportions. Several genes (Ren1, Ren2) in the renin-angiotensin system were also higher in the preterm group at $27 \mathrm{dpc}$. Ren 1 encodes for renin, which cleaves angiotensinogen to angiotensin 1 critical in blood pressure control. Angiotensin 1 is cleaved by Angiotensin-Converting Enzyme to Angiotensin II. Angiotensin II causes vasoconstriction and increases secretion of antidiuretic hormone and aldosterone, leading to increased blood pressure. These findings in the preterm mice kidney are consistent with known complications of higher blood pressures and hypertension in humans born preterm ${ }^{19,20,46,47}$. Infants born preterm have high levels of renin in serum plasma ${ }^{48}$, and many develop neonatal hypertension. The role of disrupted angiogenesis within the kidney is also consistent with studies in preterm baboons, which indicated poor glomerular vascularization ${ }^{22}$.

Using single-cell sequencing, we also observed a decrease in the subpopulation of podocytes. This finding of a smaller subpopulation of podocytes at $20 \mathrm{dpc}$ in the preterm group is consistent with human and animal studies. A limited study of biopsies of adolescents and adults who were born preterm demonstrates decreased podocyte density and FSGS lesions ${ }^{49}$. Indeed, the urine of preterm neonates is a novel source for progenitor cells, with a higher number of podocytes in the urine of infants being treated with nonsteroidal anti-inflammatory drugs ${ }^{50,51}$. The podocytes in the superficial cortex of the preterm baboon exhibit an abnormal, immature pattern of WT1+ cells surrounding relatively undifferentiated cells $^{22}$. In the present study, the preterm mice had fewer podocytes with a disorganized pattern of WT $1^{+}$ cells in the immature bodies in the nephrogenic zone, suggesting a podocytopathy may underlie the transition from preterm birth to CKD.

In our unbiased analysis approach, the fat-soluble vitamin pathway was altered; Cyp27b1 was downregulated in the preterm group, but Cyp24a1 was upregulated. Cyp27b1 and Cyp24a 1 encode members of the cytochrome P450 superfamily of enzymes that play a role in vitamin D metabolism. Cyp27b1 encodes for the enzyme 1a-hydroxylase, increasing activated vitamin D. Cyp24a 1 encodes for 24-hydroxylase, which inactivates vitamin D. Downregulation of 1a-hydroxylase and upregulation of 24hydroxylase in our preterm group indicates a reduced level of biologically active vitamin $D$. Vitamin $D$ has a significant role in organogenesis; it plays a role in bone metabolism and maintenance of calcium and phosphorus homeostasis, and has broad functions in cardiovascular health, autoimmune response, and insulin secretion ${ }^{52-55}$. Vitamin $D$ deficiency is prevalent in preterm neonates because the fetus cannot produce vitamin D. In preterm neonates, vitamin D deficiency has been associated with respiratory distress syndrome ${ }^{56}$, and predicts respiratory morbidity ${ }^{57}$. Although there is little known about the effect of vitamin D deficiency on kidney development, clinical studies indicate a relationship. In a nested study within the Generation R study, investigators demonstrate a correlation between higher maternal 25hydroxyvitamin $\mathrm{D}$ and lower childhood estimated glomerular filtration rate ${ }^{58}$. In preclinical studies in 
rodents, supplementation of vitamin D appears to reduce nephrogenesis, suggesting a role for vitamin Ddependent suppression of angiotensin II. Maternal vitamin D deficiency is associated with delayed glomerular maturation ${ }^{59}$, but it is unclear if these nephrons have any functional impairment. A delicate balance of vitamin $D$ activity is likely needed for nephrogenesis, and further work is required to define the role of vitamin $D$ in the overall kidney health of preterm neonates.

Another perturbation of the fat-soluble vitamin pathway in the preterm group was the downregulation of Crabp1 (encoding for cellular retinoic acid-binding protein-1). Vitamin A regulation is complex. The majority of the biologic functions ${ }^{60}$ of this pathway are accomplished by retinoic acid. There is evidence that upregulation of Crabp 1 can lower intracellular retinoic acid concentrations to reduce differentiation of the cell, modulate the metabolism of retinoic acid to 4-oxorentinoic acid, and increase the movement of the retinoic acid to the nucleus to interact with retinoic acid receptor initiating gene transcription 61,62 . The downregulation of Crabp 1 in the preterm mice may cause decreased retinol signaling and contribute to decreased progenitor cell self-renewal ${ }^{63}$. The vitamin A pathway also has an impact on kidney development. There is a clear role of retinol deficiency in the developing kidney and a strong correlation between plasma retinol levels and nephron number. Maternal vitamin A deficiency has been associated with renal hypoplasia ${ }^{64,65}$. Retinoic acid acts on mesenchymal cells to release branching morphogens and induce tubulogenesis ${ }^{66-70}$. Supplementation of vitamin $A$ has also been associated with an increased glomerular number in animal models of intrauterine growth restriction. Beyond the kidney, the vitamin A pathway plays an essential role in organogenesis and development, with significant deficiencies leading to fetal death and multiple congenital disabilities ${ }^{71}$. Similar to vitamin D, vitamin A deficiency has also been reported to be common in preterm neonates. Human and mice studies have also shown the association between vitamin A deficiency and poor lung outcomes ${ }^{72,73}$. Supplementation with vitamin A improved lung outcomes in these preterm neonates ${ }^{74}$.

This study uncovered a few unique perturbations in genes associated with proximal tubule, including Pdzk11, Gsta2, and Slc34a1which were significantly lower in the preterm group compared to the term group at $27 \mathrm{dpc}$. Both $P d z k 1$ and S/c34a 1 are important in phosphorus reabsorption. $P d z k 1$ encodes for a scaffolding protein for renal apical transporters such as uric acid and phosphorus reabsorption ${ }^{75}$. S/c34a 1 encodes for a sodium-dependent phosphate transporter in the brush border of the proximal tubule. Gsta2 encodes for an enzyme glutathione S-transferase A2 that is involved in the detoxification of electrophilic compounds. The decreased expression of these genes may also play a role in the development of acute kidney injury (AKI). In a murine model of sepsis, down-regulation of sodium phosphate type $2 \mathrm{a}$ transporter in the proximal tubule correlated with evidence of $\mathrm{AKI}^{76}$. Severe preterm birth is commonly associated with AKI in preterm neonates ${ }^{8}$, and most preterm neonates are exposed to proximal tubule-specific nephrotoxins such as gentamicin ${ }^{51}$. Interestingly, variants of S/c34a 1 have been associated with chronic kidney disease in genome-wide association studies ${ }^{77}$.

Unexpectedly, the pathway of immune cells was higher in the preterm group as compared to the term group, but none of the independent components reached statistical significance. Previous studies have 
demonstrated global alterations in both innate and adaptive immune cell populations in preterm rats ${ }^{78,79}$ and pigs ${ }^{80-82}$, but no studies have examined the immune cell populations within the kidney. Further work is necessary to determine if these immune cells are resident cells or just captured in the vasculature and what role, if any, they play in kidney development.

There are limitations of this study, including the availability of scRNA-seq data of neonatal mouse kidneys. Testing expression profile alterations on each nephron progenitor subpopulation using scRNAseq could provide further insights to understand the effect of preterm birth on kidney development.

Together, our data provide the first insights into gene dysregulation and changes in proportions of cell subtypes that may contribute to kidney disease and risk of hypertension in those born preterm. The preterm mouse model provides a means to dissect the molecular mechanism underlying premature cessation of nephrogenesis to develop targeted strategies to reduce the risk of CKD. This environmental model is unique because the nephrotoxin exposures and other insults common in preterm humans are absent, isolating the effect of preterm birth. Our study demonstrates dysregulation of potentially modifiable pathways in the preterm kidney, including alterations in vitamin metabolism. Further work is required to decipher the molecular mechanisms that influence the cessation of nephrogenesis to safely apply new tools to extend the duration of nephrogenesis in preterm neonates.

\section{Declarations}

\section{Funding:}

Project funded by 3P50DK096373 (Pilot Project) and ASN Carl W. Gottschalk Research Scholar Grant JRC and KMB are funded by NIH/NIDDK: R01DK111861 and R01DK110622.

JRC is funded by $1 \mathrm{U} 34 \mathrm{DK} 117128$.

SED is funded by NIH R01 DK114050

\section{Author Contribution Statement:}

JRC, AC, KdR planned and carried out the experiments. MS and MC performed the data analysis and all authors contributed to the interpretation of the results. AC, MS, KR and JRC wrote the first draft of the manuscript. All authors provided critical feedback and helped shape the research and analysis. All authors reviewed and approved the final manuscript.

\section{Disclosures:}

KJR is a site investigator for unrelated clinical trials supported by Advicenne and Travere Therapeutics. KMB and JRC are co-owners of Sindri Technologies, LLC. 
KMB is co-owner of Nephrodiagnostics, LLC, and XN Biotechologies, LLC.

KMB has a research agreement with Janssen Pharmaceutical.

\section{References}

1 Stoll, B. J. et al. Neonatal outcomes of extremely preterm infants from the NICHD Neonatal Research Network. Pediatrics 126, 443-456, doi:10.1542/peds.2009-2959 (2010).

$2 \quad$ Horbar, J. D. et al. Mortality and neonatal morbidity among infants 501 to 1500 grams from 2000 to 2009. Pediatrics 129, 1019-1026, doi:10.1542/peds.2011-3028 (2012).

3 Hinchliffe, S. A., Sargent, P. H., Howard, C. V., Chan, Y. F. \& van Velzen, D. Human intrauterine renal growth expressed in absolute number of glomeruli assessed by the disector method and Cavalieri principle. Lab Invest 64, 777-784 (1991).

4 Sutherland, M. R. et al. Accelerated Maturation and Abnormal Morphology in the Preterm Neonatal Kidney. Journal of the American Society of Nephrology 22, 1365-1374, doi:10.1681/asn.2010121266 (2011).

5 Osathanondh, V. \& Potter, E. L. DEVELOPMENT OF HUMAN KIDNEY AS SHOWN BY MICRODISSECTION. III. FORMATION AND INTERRELATIONSHIP OF COLLECTING TUBULES AND NEPHRONS. Arch Pathol 76, 290-302 (1963).

6 Lavery, A. P. et al. Urinary NGAL in Premature Infants. Pediatric Research 64, 423-428, doi:10.1203/pdr.0b013e318181b3b2 (2008).

7 Patzer, L. Nephrotoxicity as a cause of acute kidney injury in children. Pediatric Nephrology 23, 2159-2173, doi:10.1007/s00467-007-0721-x (2008).

8 Jetton, J. G. et al. Incidence and outcomes of neonatal acute kidney injury (AWAKEN): a multicentre, multinational, observational cohort study. Lancet Child Adolesc Health 1, 184-194, doi:10.1016/S2352-4642(17)30069-X (2017).

9 Charlton, J. R. et al. Late onset neonatal acute kidney injury: results from the AWAKEN Study. Pediatric Research 85, 339-348, doi:10.1038/s41390-018-0255-x (2019).

10 Crump, C., Sundquist, J., Winkleby, M. A. \& Sundquist, K. Preterm birth and risk of chronic kidney disease from childhood into mid-adulthood: national cohort study.

11 Hsu, C. W., Yamamoto, K. T., Henry, R. K., De Roos, A. J. \& Flynn, J. T. Prenatal Risk Factors for Childhood CKD. Journal of the American Society of Nephrology 25, 2105-2111, doi:10.1681/asn.2013060582 (2014). 
12 White, S. L. et al. Is Low Birth Weight an Antecedent of CKD in Later Life? A Systematic Review of Observational Studies. American Journal of Kidney Diseases 54, 248-261, doi:10.1053/j.ajkd.2008.12.042 (2009).

13 Hoogenboom, L. A., Wolfs, T. G. A. M., Hütten, M. C., Peutz-Kootstra, C. J. \& Schreuder, M. F. Prematurity, perinatal inflammatory stress, and the predisposition to develop chronic kidney disease beyond oligonephropathy. Pediatric Nephrology, doi:10.1007/s00467-020-04712-2 (2020).

14 Khalsa, D. D. K., Beydoun, H. A. \& Carmody, J. B. Prevalence of chronic kidney disease risk factors among low birth weight adolescents. Pediatric Nephrology 31, 1509-1516, doi:10.1007/s00467-016-33847 (2016).

15 Bonamy, A.-K. E. et al. Preterm Birth Contributes to Increased Vascular Resistance and Higher Blood Pressure in Adolescent Girls. Pediatric Research 58, 845-849, doi:10.1203/01.pdr.0000181373.29290.80 (2005).

16 Bonamy, A.K. E., Kallen, K. \& Norman, M. High Blood Pressure in 2.5-Year-Old Children Born Extremely Preterm. PEDIATRICS 129, e1199-e1204, doi:10.1542/peds.2011-3177 (2012).

17 Lazdam, M. et al. Elevated Blood Pressure in Offspring Born Premature to Hypertensive Pregnancy. Hypertension 56, 159-165, doi:10.1161/hypertensionaha.110.150235 (2010).

18 Stelloh, C. et al. Prematurity in mice leads to reduction in nephron number, hypertension, and proteinuria. Translational research : the journal of laboratory and clinical medicine 159, 80-89, doi:10.1016/j.trsl.2011.10.004 (2012).

19 South, A. M. et al. Renal function and blood pressure are altered in adolescents born preterm. Pediatric Nephrology 34, 137-144, doi:10.1007/s00467-018-4050-z (2019).

20 South, A. M. et al. Association between preterm birth and the renin-angiotensin system in adolescence. Journal of Hypertension 36, 2092-2101, doi:10.1097/hjh.0000000000001801 (2018).

21 Rodríguez, M. M. et al. Histomorphometric Analysis of Postnatal Glomerulogenesis in Extremely Preterm Infants. Pediatric and Developmental Pathology 7, 17-25, doi:10.1007/s10024-003-3029-2 (2004).

22 Gubhaju, L. et al. Is nephrogenesis affected by preterm birth? Studies in a non-human primate model. Am J Physiol Renal Physio/ 297, F1668-1677, doi:10.1152/ajprenal.00163.2009 (2009).

23 Hodgin, J. B., Rasoulpour, M., Markowitz, G. S. \& D'Agati, V. D. Very Low Birth Weight is a Risk Factor for Secondary Focal Segmental Glomerulosclerosis. Clinical Journal of the American Society of Nephrology 4, 71-76, doi:10.2215/cjn.01700408 (2009). 
24 Gubhaju, L. \& Black, M. J. The Baboon as a Good Model for Studies of Human Kidney Development. Pediatric Research 58, 505-509, doi:10.1203/01.pdr.0000179397.20862.73 (2005).

25 Minuth, W. W. The rabbit nephrogenic zone in culture: past, present and future as a model to investigate causes of impaired nephrogenesis.

26 Mccarthy, R. et al. Mouse models of preterm birth: suggested assessment and reporting guidelinest. Biology of Reproduction, doi:10.1093/biolre/ioy109 (2018).

27 Grases-Pintó, B. et al. A Preterm Rat Model for Immunonutritional Studies. Nutrients 11, 999, doi:10.3390/nu11050999 (2019).

28 Wang, L., Wang, S. \& Li, W. RSeQC: quality control of RNA-seq experiments. Bioinformatics 28, 2184-2185, doi:10.1093/bioinformatics/bts356 (2012).

29 Dobin, A. et al. STAR: ultrafast universal RNA-seq aligner. Bioinformatics 29, 15-21, doi:10.1093/bioinformatics/bts635 (2013).

30 Love, M. I., Huber, W. \& Anders, S. Moderated estimation of fold change and dispersion for RNAseq data with DESeq2. Genome Biology 15, doi:10.1186/s13059-014-0550-8 (2014).

31 Adam, M., Potter, A. S. \& Potter, S. S. Psychrophilic proteases dramatically reduce single-cell RNAseq artifacts: a molecular atlas of kidney development. Development 144, 3625-3632, doi:10.1242/dev.151142 (2017).

32 Newman, A. M. et al. Determining cell type abundance and expression from bulk tissues with digital cytometry. Nature Biotechnology 37, 773-782, doi:10.1038/s41587-019-0114-2 (2019).

33 Satija, R., Farrell, J. A., Gennert, D., Schier, A. F. \& Regev, A. Spatial reconstruction of single-cell gene expression data. Nature Biotechnology 33, 495-502, doi:10.1038/nbt.3192 (2015).

34 Trapnell, C. et al. The dynamics and regulators of cell fate decisions are revealed by pseudotemporal ordering of single cells. Nature Biotechnology 32, 381-386, doi:10.1038/nbt.2859 (2014).

35 Martinez, Maria F., Martini, Alexandre G., Sequeira-Lopez, M. Luisa S. \& Gomez, R. A. Ctcf is required for renin expression and maintenance of the structural integrity of the kidney. Clinical Science 134, 1763-1774, doi:10.1042/cs20200184 (2020).

36 Yu, G., Wang, L.-G., Han, Y. \& He, Q.-Y. clusterProfiler: an R Package for Comparing Biological Themes Among Gene Clusters. OMICS: A Journal of Integrative Biology 16, 284-287, doi:10.1089/omi.2011.0118 (2012).

37 Lescher, B., Haenig, B. \& Kispert, A. sFRP-2 is a target of the Wnt-4 signaling pathway in the developing metanephric kidney. Dev Dyn 213, 440-451, doi:10.1002/(sici)1097- 
0177(199812)213:4<440::aid-aja9>3.0.co;2-6 (1998).

38 Brown, A. C., Muthukrishnan, S. D. \& Oxburgh, L. A synthetic niche for nephron progenitor cells. Dev Cell 34, 229-241, doi:10.1016/j.devcel.2015.06.021 (2015).

39 Jahnukainen, T., Chen, M., Berg, U. \& Celsi, G. Antenatal glucocorticoids and renal function after birth. Seminars in Neonatology 6, 351-355, doi:10.1053/siny.2001.0070 (2001).

40 Haycock. Development of glomerular filtration and tubular sodium reabsorption in the human fetus and newborn. British Journal of Urology 81, 33-38, doi:10.1046/j.1464-410x.1998.0810s2033.x (1998).

41 Hartman, H. A., Lai, H. L. \& Patterson, L. T. Cessation of renal morphogenesis in mice. Developmental Biology 310, 379-387, doi:10.1016/j.ydbio.2007.08.021 (2007).

42 Short, M., Kieran et al. Global Quantification of Tissue Dynamics in the Developing Mouse Kidney. Developmental Cell 29, 188-202, doi:10.1016/j.devcel.2014.02.017 (2014).

43 Short, K. M. \& Smyth, I. M. The contribution of branching morphogenesis to kidney development and disease. Nature Reviews Nephrology 12, 754-767, doi:10.1038/nrneph.2016.157 (2016).

44 Kobayashi, A. et al. Six2 Defines and Regulates a Multipotent Self-Renewing Nephron Progenitor Population throughout Mammalian Kidney Development. Cell Stem Cel/ 3, 169-181, doi:https://doi.org/10.1016/j.stem.2008.05.020 (2008).

45 Boyle, S. et al. Fate mapping using Cited1-CreERT2 mice demonstrates that the cap mesenchyme contains self-renewing progenitor cells and gives rise exclusively to nephronic epithelia. Developmental Biology 313, 234-245, doi:https://doi.org/10.1016/j.ydbio.2007.10.014 (2008).

46 Nishizaki, N. et al. Increased urinary angiotensinogen is an effective marker of chronic renal impairment in very low birth weight children. Clinical and Experimental Nephrology 18, 642-648, doi:10.1007/s10157-013-0896-3 (2014).

47 Khalsa, D. D., Beydoun, H. A. \& Carmody, J. B. Prevalence of chronic kidney disease risk factors among low birth weight adolescents. Pediatric nephrology (Berlin, Germany) 31, 1509-1516, doi:10.1007/s00467-016-3384-7 [doi] (2016).

48 Stephenson, T. J., Broughton Pipkin, F. \& Elias-Jones, A. C. Factors influencing plasma renin and renin substrate in premature infants. Archives of Disease in Childhood 66, 1150-1154, doi:10.1136/adc.66.10_spec_no.1150 (1991).

49 Ikezumi, Y. et al. Low Birthweight and Premature Birth Are Risk Factors for Podocytopenia and Focal Segmental Glomerulosclerosis. American Journal of Nephrology 38, 149-157, doi:10.1159/000353898 (2013). 
50 Arcolino, F. O. et al. Urine of Preterm Neonates as a Novel Source of Kidney Progenitor Cells. Journal of the American Society of Nephrology 27, 2762-2770, doi:10.1681/asn.2015060664 (2016).

51 Kent, A. L. et al. Renal Glomeruli and Tubular Injury Following Indomethacin, Ibuprofen, and Gentamicin Exposure in a Neonatal Rat Model. Pediatric Research 62, 307-312, doi:10.1203/pdr.0b013e318123f6e3 (2007).

52 Di Rosa, M., Malaguarnera, M., Nicoletti, F. \& Malaguarnera, L. Vitamin D3: a helpful immunomodulator. Immunology 134, 123-139, doi:10.1111/j.1365-2567.2011.03482.x (2011).

53 Fukumoto, S. Phosphate metabolism and vitamin D. BoneKEy Reports 3, doi:10.1038/bonekey.2013.231 (2014).

54 Danik, J. S. \& Manson, J. E. Vitamin D and Cardiovascular Disease. Current Treatment Options in Cardiovascular Medicine 14, 414-424, doi:10.1007/s11936-012-0183-8 (2012).

55 Hoseini, S. A., Aminorroaya, A., Iraj, B. \& Amini, M. The effects of oral vitamin D on insulin resistance in pre-diabetic patients. J Res Med Sci 18, 47-51 (2013).

$56 \mathrm{Kim}, \mathrm{I}$. et al. Association between vitamin D level at birth and respiratory morbidities in very-lowbirth-weight infants. Korean Journal of Pediatrics 62, 166-172, doi:10.3345/kjp.2018.06632 (2019).

57 Golan-Tripto, I. et al. The effect of vitamin D administration on vitamin D status and respiratory morbidity in late premature infants. Pediatric Pulmonology, doi:10.1002/ppul.25006 (2020).

58 Maka, N. et al. Vitamin D deficiency during pregnancy and lactation stimulates nephrogenesis in rat offspring. Pediatric Nephrology 23, 55-61, doi:10.1007/s00467-007-0641-9 (2008).

59 Nascimento, F. A. M., Ceciliano, T. C., Aguila, M. B. \& Mandarim-De-Lacerda, C. A. Maternal Vitamin D Deficiency Delays Glomerular Maturity in F1 and F2 Offspring. PLoS ONE 7, e41740, doi:10.1371/journal.pone.0041740 (2012).

60 Mark, M., Ghyselinck, N. B. \& Chambon, P. FUNCTION OF RETINOID NUCLEAR RECEPTORS: Lessons from Genetic and Pharmacological Dissections of the Retinoic Acid Signaling Pathway During Mouse Embryogenesis. Annual Review of Pharmacology and Toxicology 46, 451-480, doi:10.1146/annurev.pharmtox.46.120604.141156 (2006).

61 Wei, L.-N., Chang, L. \& Hu, X. Studies of the type I cellular retinoic acid-binding protein mutants and their biological activities. Molecular and Cellular Biochemistry 200, 69-76, doi:10.1023/a:1006906415388 (1999).

62 Lane, M. A. et al. LIF removal increases CRABPI and CRABPII transcripts in embryonic stem cells cultured in retinol or 4-oxoretinol. Molecular and Cellular Endocrinology 280, 63-74, doi:10.1016/j.mce.2007.09.010 (2008). 
63 Chen, L. \& Khillan, J. S. A novel signaling by vitamin A/retinol promotes self renewal of mouse embryonic stem cells by activating PI3K/Akt signaling pathway via insulin-like growth factor-1 receptor. Stem Cells 28, 57-63, doi:10.1002/stem.251 (2010).

64 Goodyer, P. et al. Effects of maternal vitamin A status on kidney development: a pilot study. Pediatric Nephrology 22, 209-214, doi:10.1007/s00467-006-0213-4 (2007).

65 Cain, J. E., Di Giovanni, V., Smeeton, J. \& Rosenblum, N. D. Genetics of Renal Hypoplasia: Insights Into the Mechanisms Controlling Nephron Endowment. Pediatric Research 68, 91-98, doi:10.1203/pdr.0b013e3181e35a88 (2010).

66 Gandhi, D. et al. Retinoid Signaling in Progenitors Controls Specification and Regeneration of the Urothelium. Developmental Cel/ 26, 469-482, doi:10.1016/j.devcel.2013.07.017 (2013).

67 Batourina, E. et al. Vitamin A controls epithelial/mesenchymal interactions through Ret expression. Nature Genetics 27, 74-78, doi:10.1038/83792 (2001).

68 Humes, H. D. \& Cieslinski, D. A. Interaction between growth factors and retinoic acid in the induction of kidney tubulogenesis in tissue culture. Experimental Cell Research 201, 8-15, doi:10.1016/0014-4827(92)90342-6 (1992).

69 Vilar, J., Gilbert, T., Moreau, E. \& Merlet-Bénichou, C. Metanephros organogenesis is highly stimulated by vitamin A derivatives in organ culture. Kidney International 49, 1478-1487, doi:10.1038/ki.1996.208 (1996).

70 Rosselot, C. et al. Non-cell-autonomous retinoid signaling is crucial for renal development. Development 137, 283-292, doi:10.1242/dev.040287 (2010).

71 Wilson, J. G., Roth, C. B. \& Warkany, J. An analysis of the syndrome of malformations induced by maternal vitamin a deficiency. Effects of restoration of vitamin a at various times during gestation. American Journal of Anatomy 92, 189-217, doi:10.1002/aja.1000920202 (1953).

72 Chen, F. et al. Prenatal retinoid deficiency leads to airway hyperresponsiveness in adult mice. Journal of Clinical Investigation 124, 801-811, doi:10.1172/jci70291 (2014).

73 Timoneda, J. et al. Vitamin A Deficiency and the Lung. Nutrients 10, 1132, doi:10.3390/nu10091132 (2018).

74 Checkley, W. et al. Maternal Vitamin A Supplementation and Lung Function in Offspring. New England Journal of Medicine 362, 1784-1794, doi:10.1056/nejmoa0907441 (2010).

75 Endou, H. \& Anzai, N. Urate transport across the apical membrane of renal proximal tubules. Nucleosides Nucleotides Nucleic Acids 27, 578-584, doi:10.1080/15257770802136024 (2008). 
76 Kamimoto, M., Mizuno, S., Ohnishi, H. \& Mizuno-Horikawa, Y. Type 2a sodium-phosphate cotransporter serves as a histological predictor of renal dysfunction and tubular apical damage in the kidneys of septic mice. Biomed Res 30, 251-258, doi:10.2220/biomedres.30.251 (2009).

77 Wagner, C. A., Rubio-Aliaga, I. \& Hernando, N. Renal phosphate handling and inherited disorders of phosphate reabsorption: an update. Pediatric Nephrology 34, 549-559, doi:10.1007/s00467-017-3873-3 (2019).

78 Grases-Pinto, B. et al. A Preterm Rat Model for Immunonutritional Studies. Nutrients 11, doi:10.3390/nu11050999 (2019).

79 Grases-Pinto, B. et al. Leptin and EGF Supplementation Enhance the Immune System Maturation in Preterm Suckling Rats. Nutrients 11, doi:10.3390/nu11102380 (2019).

80 Arrindell, E. L., Jr. et al. Lung volume recruitment in a preterm pig model of lung immaturity. Am J Physiol Lung Cell Mol Physio/ 309, L1088-1092, doi:10.1152/ajplung.00292.2015 (2015).

81 Caminita, F. et al. A preterm pig model of lung immaturity and spontaneous infant respiratory distress syndrome. Am J Physiol Lung Cell Mol Physio/ 308, L118-129, doi:10.1152/ajplung.00173.2014 (2015).

82 Sangild, P. T. et al. Invited review: the preterm pig as a model in pediatric gastroenterology. J Anim Sci 91, 4713-4729, doi:10.2527/jas.2013-6359 (2013).

\section{Figures}

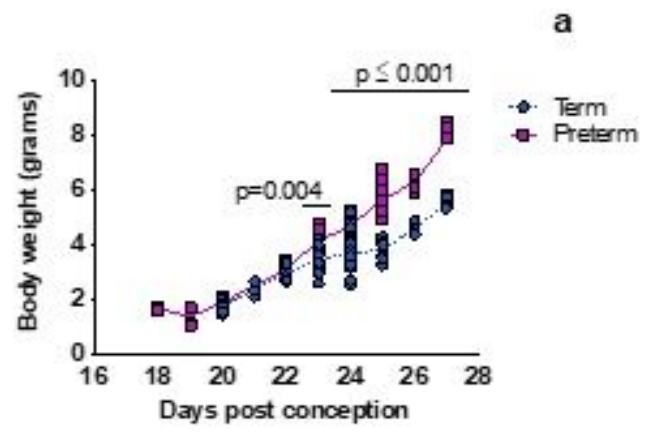

c

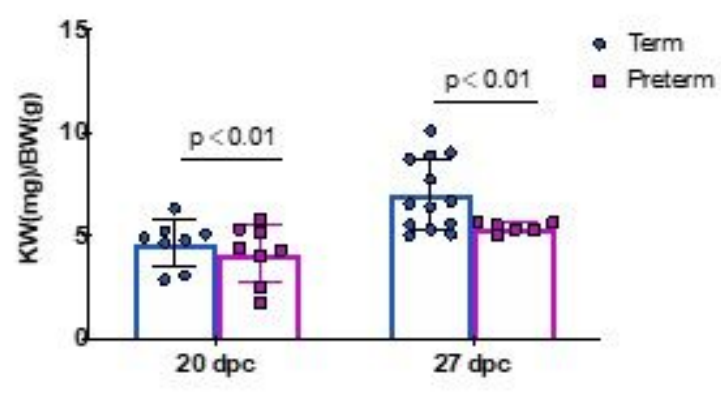

b
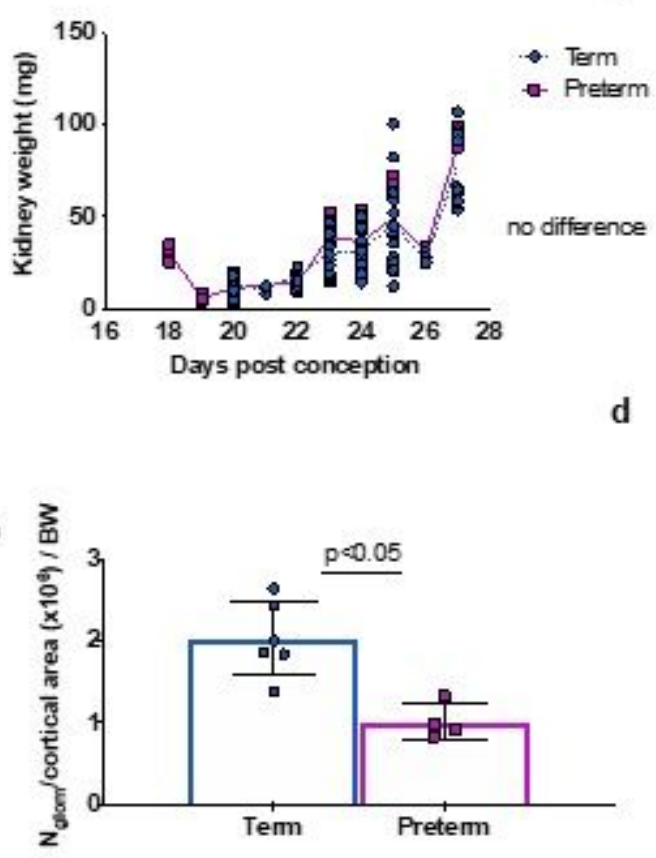


\section{Figure 1}

Body weight, kidney weight and glomerular density. Beginning at $23 \mathrm{dpc}$, the weight of the animals in the preterm group was greater than that of the term group (a). There was no difference in the absolute kidney weight between the preterm and term groups (b). When kidney weight was normalized to body weight, the preterm group had smaller kidneys at both 20 and $27 \mathrm{dpc}$ (c). Glomerular density at $27 \mathrm{dpc}$ was lower in the preterm group than in the term group (d).

\section{Lotus lectin}
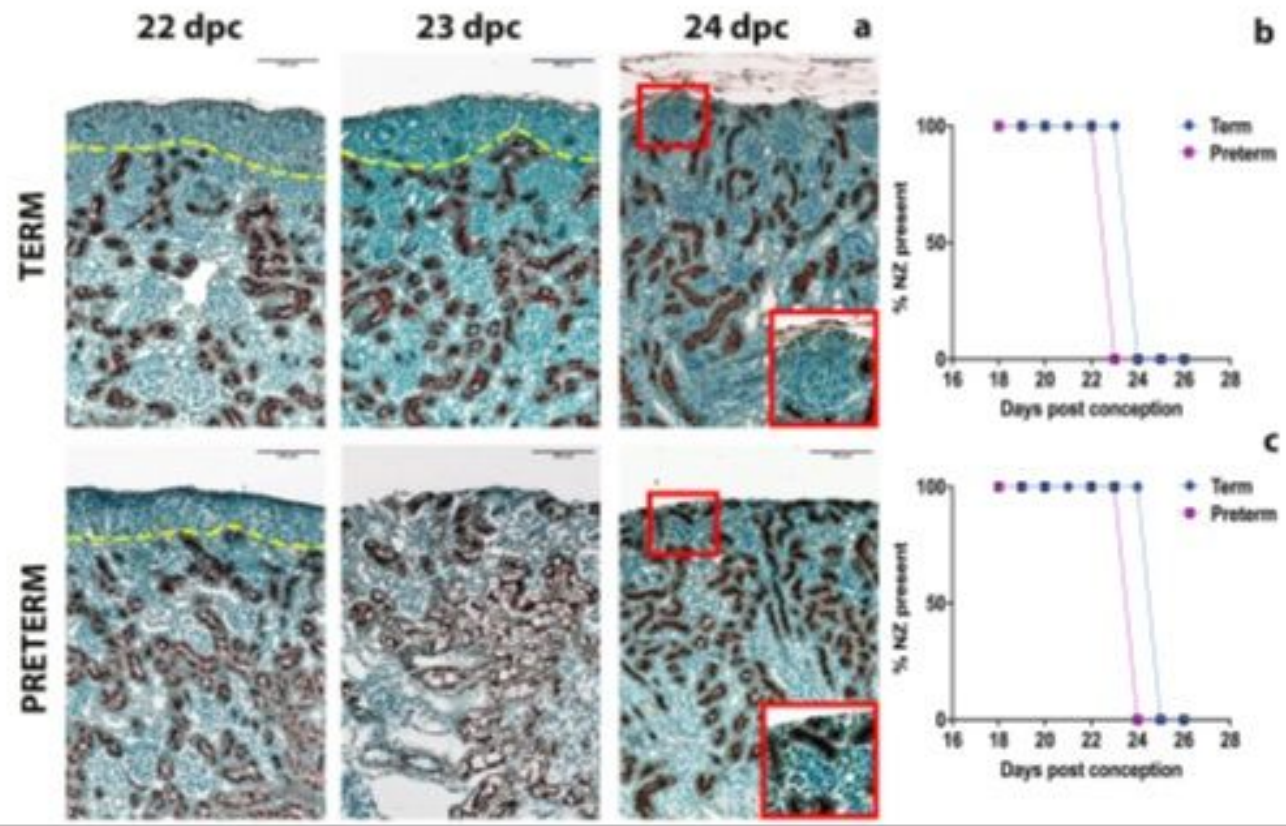

d
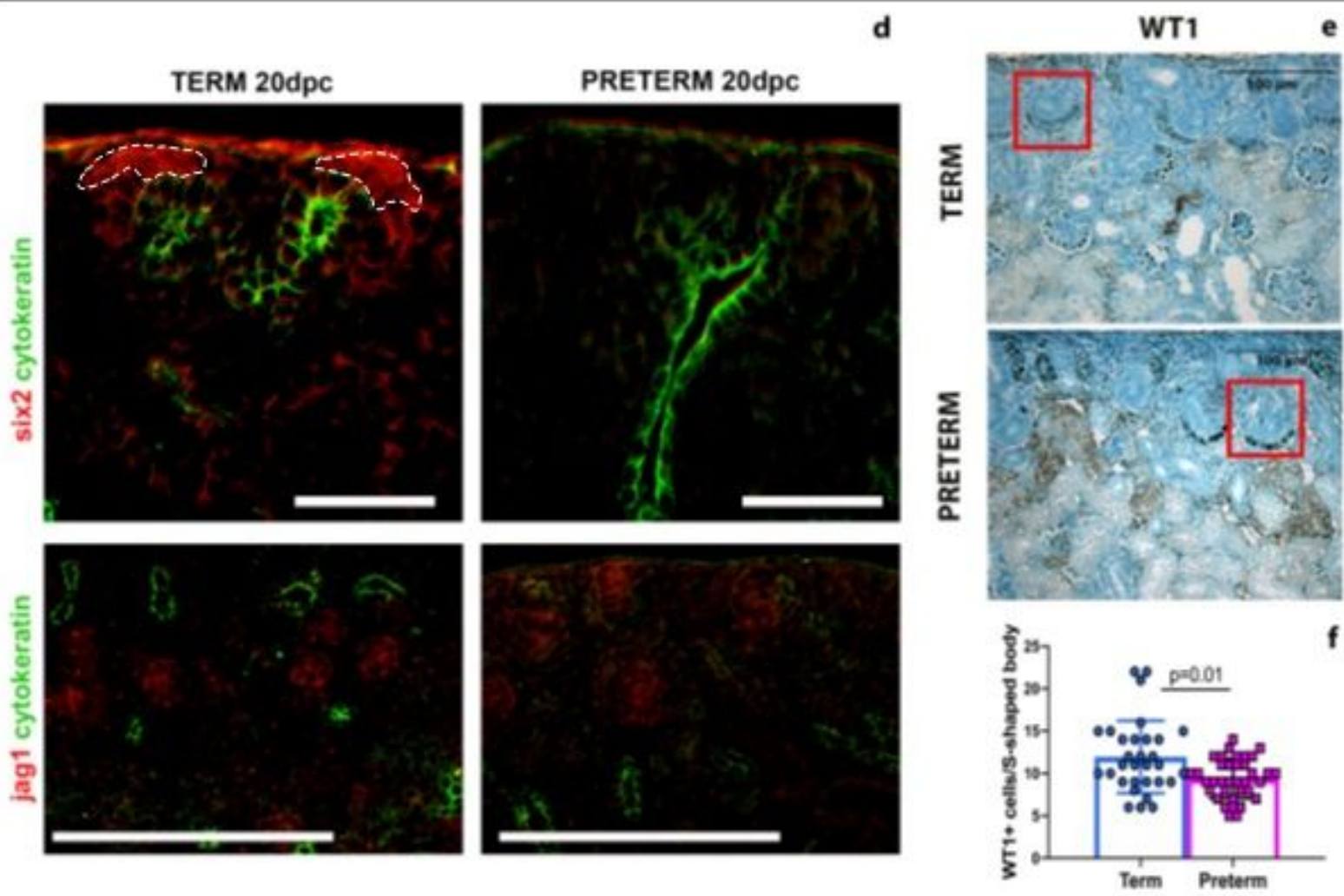


\section{Figure 2}

Preterm mice have a shorter duration of nephrogenesis. The nephrogenic zone is outlined by the yellow line in the LTL-stained kidneys (a). The nephrogenic zone was depleted when there were no longer cap mesenchymal cells and when LTL+ cells reach the top of the capsule. The nephrogenic zone is absent in the preterm cohort one day before the term group (a). The same LTL-stained tissue was used to determine the presence of immature structures (vesicles, comma- and S-shaped bodies), which were not not detectable in preterm animals one day before than in the term cohort (c). In the red square in panel a of a term mouse kidney at $24 \mathrm{dpc}$, the S-shaped body indicates the presence of immature structures at this time point in the term group. However, the preterm group lacks these immature structures. Representative pictures of Six2 and Jag1 staining showing the loss of the Six2 protein expression in the nephrogenic zone and increase of the Jag1 expressed in the nephrogenic zone, consistent with early differentiation of the preterm group at $20 \mathrm{dpc}(\mathrm{d})$. Panel (e) is a representative image of WT-1 staining within the kidney of a term and preterm mouse at $20 \mathrm{dpc}$. Within the red box, eight WT-1+ cells can be counted, whereas, in panel e, there are only 6 WT $1+$ cells. Interestingly, under identical conditions, the WT1+ cells consistently appeared darker in the preterm group as compared to the term group, which may be related to a more advanced state of differentiation. Overall, the term group had more WT1+ cells than the preterm group at $20 \mathrm{dpc}(\mathrm{f})$. Scale bars in a and e $=100 \mu \mathrm{m}$. Scale bars in d: Six $2 /$ cytokeratin $=10 \mu \mathrm{m}$; in Jag1/cytokeratin= $200 \mu \mathrm{m}$. 
$20 \mathrm{dpc}$

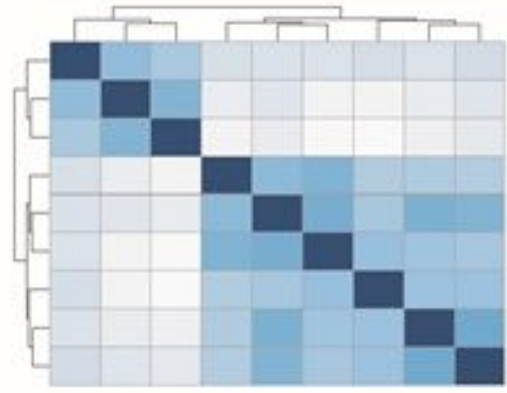

$20 \mathrm{dpc}$

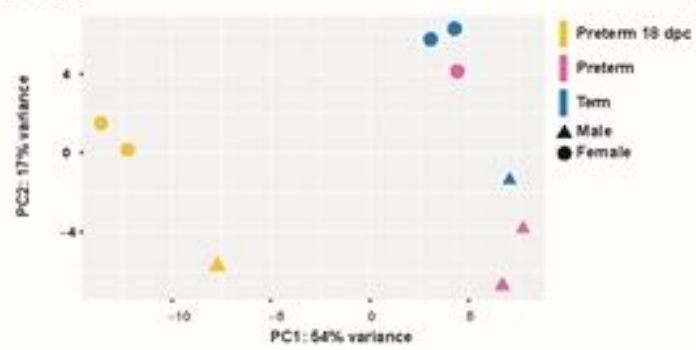

$20 \mathrm{dpc}$

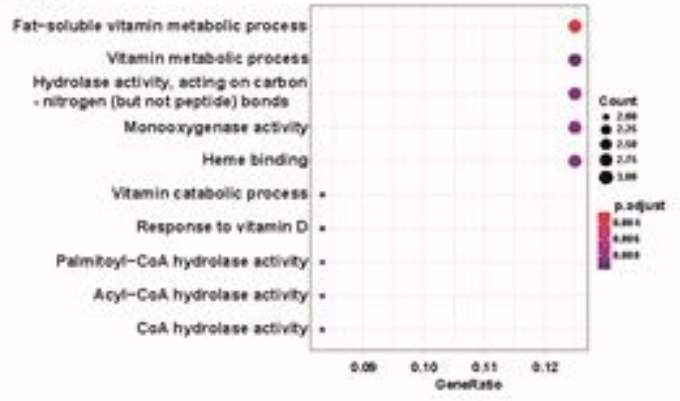

a

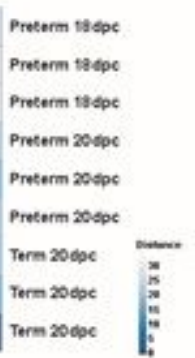

b

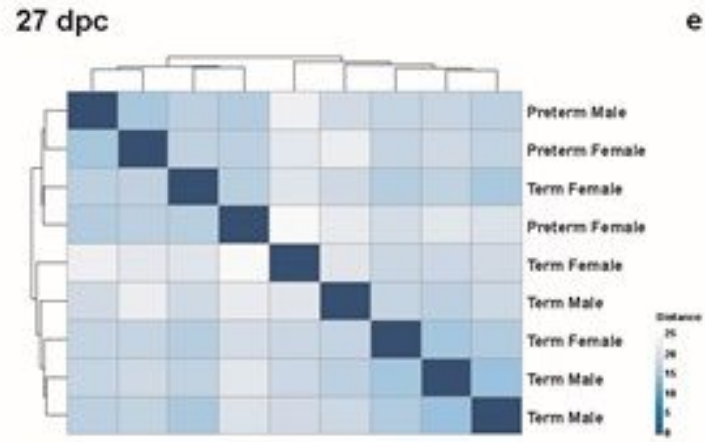

$27 \mathrm{dpc}$

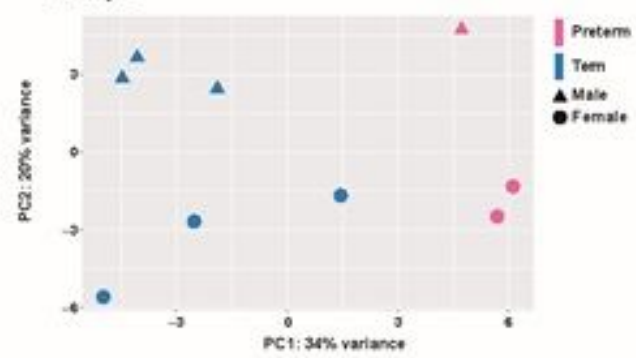

$27 \mathrm{dpc}$

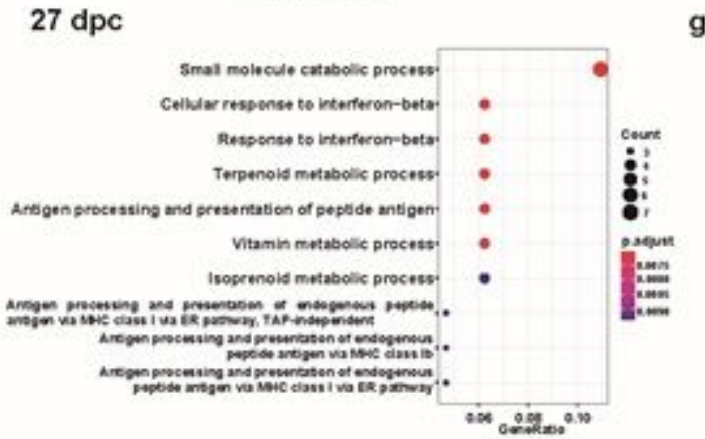

$20 \mathrm{dpc}$

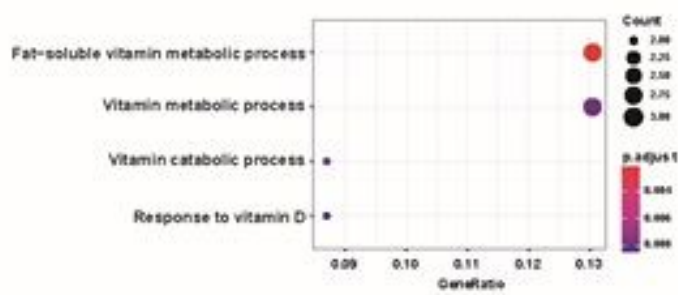

d

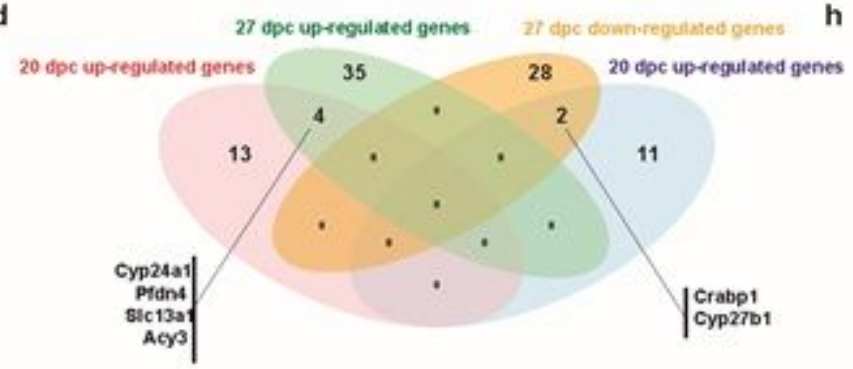

\section{Figure 3}

Preterm birth results in early transcriptional alterations in the kidney. (a) Hierarchical clustering analysis on RNA-seq data revealed the expression profiles clustering into three groups at the early time point (18 and $20 \mathrm{dpc}$ ), with apparent differences between the $18 \mathrm{dpc}$ animals from those at $20 \mathrm{dpc}$. (b) A principal component analysis also showed clear dissociations between $18 \mathrm{dpc}$ and $20 \mathrm{dpc}$ expression profiles. Among the $20 \mathrm{dpc}$ animals, there was sex-dependent clustering. Gene Ontology (GO) enrichment analyses (c) and (d) on differentially expressed genes (DEGs) between preterm and term 20 kidneys revealed enrichment of the fat-soluble vitamin related pathways in an analysis adjusted (d) or not adjusted (c) for sex. (e) Hierarchical clustering analysis on the expression profiles of $27 \mathrm{dpc}$ animals. (f) A principal component analysis of $27 \mathrm{dpc}$ expression data also showed dissociations between preterm and 
term expression profiles and a sex-dependent clustering. (g) GO enrichment analysis on DEGs of $27 \mathrm{dpc}$ without adjusting for sex showed small catabolic processes as the top GO term. (h) A Venn diagram showed overlaps of DEGs between 20 and 27 dpc analyses. Six overlapped DEGs were identified (4 upregulated genes and 2 down-regulated genes) with the same direction of the alterations at 20 and $27 \mathrm{dpc}$.
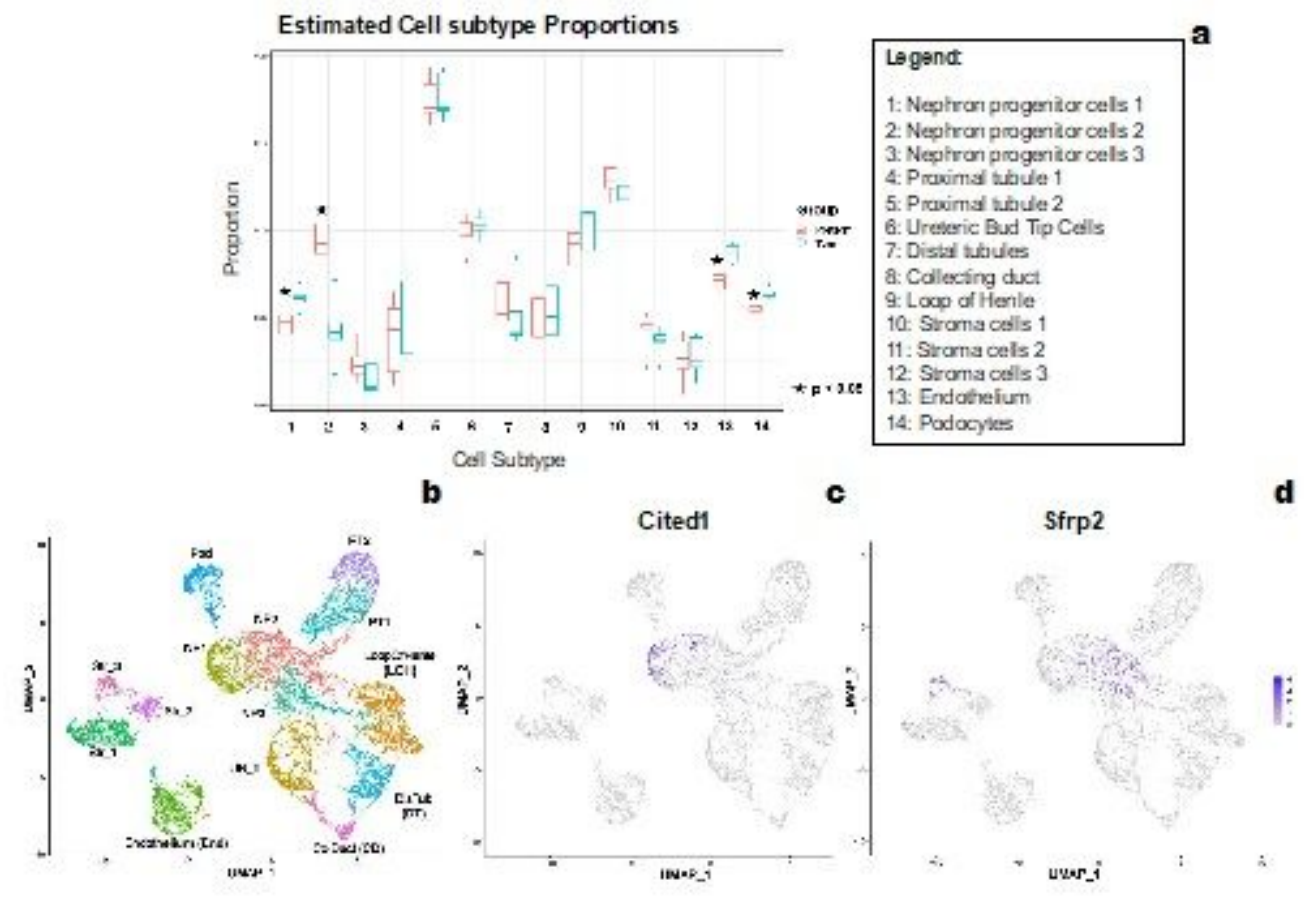

\section{Figure 4}

Preterm birth alters the cell subtype proportion of the kidney. A cell subtype proportion analysis on RNAseq data showed kidneys from the preterm group had a decreased proportion of nephron progenitor 1 cells (NP1) and an increased proportion of NP2 cells (NP). The cell proportion of endothelial cells and podocytes was also lower in the preterm group. Identified cell subtype clusters in the scRNA-seq data (b). The nephron progenitor subpopulation marker genes Cited1 and Sfrp1 were used to classified the NP subpopulations with Cited1 marking the nephron progenitor 1 cluster (NP1) (c) and Sfrp2 marking the more differentiated nephron progenitor 2 cluster (NP2) (d). *Abbreviations in part b: NP1-3: nephron progenitor cells 1-3; UB_T: ureteric bud tip cells; Str_1-3: stromal cells 1-3; Pod: podocytes; PT1-2: proximal tubule 1-2; LOH: Loop of Henle; DT: distal tubule; CD: collecting duct; End: endothelium.

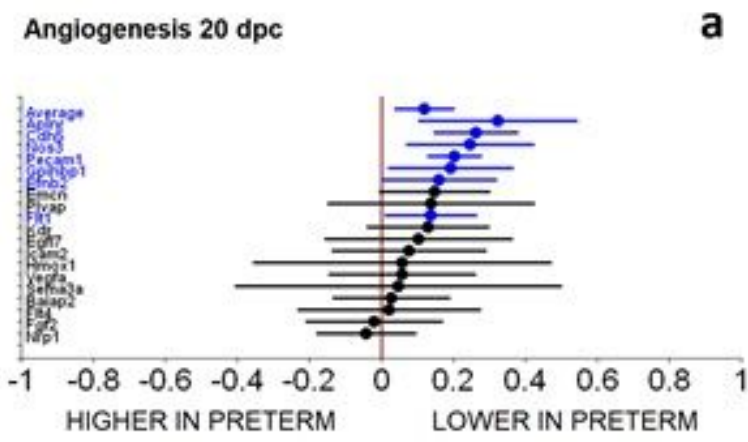

a

Immune cell designation $\mathbf{2 7} \mathrm{dpc}$

b

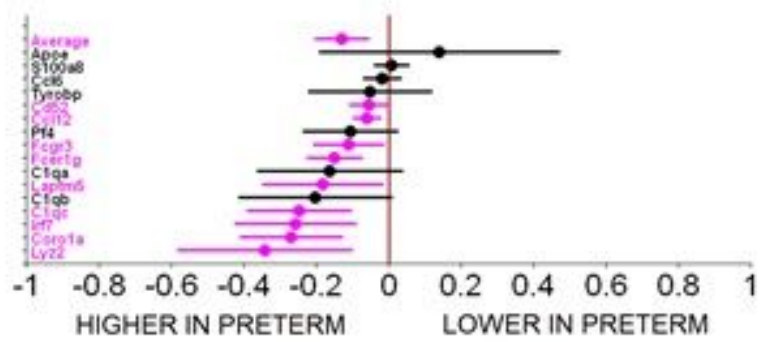

Figure 5 
Angiogenesis at $20 \mathrm{dpc}$ and immune cell designation at $27 \mathrm{dpc}$ were the top differential gene expression pathways. At $20 \mathrm{dpc}$, differential gene expression of seven (gene names highlighted in red) out of 19 genes with confidence intervals $(\mathrm{Cl})$ indicating lower gene expression in the preterm group as compared to the term group were observed in the angiogenesis pathway (Figure 5a). At $27 \mathrm{dpc}$, the category of immune cell designation showed nine of the sixteen genes that had Cl's with higher gene expression in the preterm group compared to the term group (Figure $5 b$ ).

\section{Supplementary Files}

This is a list of supplementary files associated with this preprint. Click to download.

- SciReportPremieSupplementalTables.xlsx

- SciReportPremieSupplementarydataFigureS1.docx

- SciReportSupplementaryDataFigureS2.docx

- SciReportSupplementaryDataFigureS3ad20.pdf

- SciReportSupplementaryDataFigureS3bd27.pdf

- SciReportSupplementaryDataFigureS4.docx 